\section{Senrad: An Advanced Wideband Air-Surveillance Radar}

MERRILL SKOLNIK, Life Fellow, IEEE

GEORGE LINDE, Member, IEEE

KEITH MEADS

Naval Research Laboratory

The generic characteristics and performance of an experimental long-range air-surveillance radar, known at the Naval Research Laboratory as Senrad, is described. Its distinguishing feature is that it can operate with simultaneous transmissions over a very wide bandwidth-from 850 to $1400 \mathrm{MHz}$. The technology and type of experimental radar equipment employed are discussed and examples are given of its performance capabilities obtained by means of very wideband operation. The unusually wide bandwidth of this radar allows 1) improved detection and tracking performance because of the absence of the nulls that are common in the antenna elevation radiation-pattern of a single-frequency radar; 2) moving target indication (MTI) without loss of targets due to blind speeds and without the need for multiple PRFs (pulse repetition frequencies); 3) accurate height finding with a fan-beam radar by taking advantage of the multipath time difference as a function of target height; 4) a form of limited target recognition based on high range-resolution; and 5) a reduction of the effectiveness of electronic countermeasures that can seriously degrade more narrowband radars.

Manuscript received October 6, 1999; revised January 23 and July 28, 2001; released for publication July 28, 2001.

IEEE Log No. T-AES/37/4/10984.

Refereeing of this contribution was handled by L. M. Kaplan.

Authors' address: Code 5007, Naval Research Laboratory, 4555 Overlook Ave. SW, Washington, DC 20375.

U.S. Government work not protected by U.S. copyright.

\section{INTRODUCTION}

Ever since the inception of radar in the 1930s, the surveillance of air targets has been one of its most important applications. There have been many different types of radars developed for this purpose in many different radar-frequency bands [1-7]. Improved radars have appeared over the years as a result of the appearance of new technology or new requirements. This paper describes the generic characteristics and performance of a long-range (nominally $200 \mathrm{nmi}$ ) air-surveillance radar concept whose key feature is that it utilizes an unusually wide frequency band to radiate at simultaneous multiple frequencies. It is described here as a long-range air-surveillance radar that operates over water, but the basic technology is applicable to other types of air-surveillance systems, as well as for air-traffic control. The objective of the radar development program described here, which was called Senrad, was to investigate the many advantages of operating a radar simultaneously in two radar bands with very wide bandwidth. Compared with the conventional narrowband air-surveillance radar, these advantages include improved detection, tracking, and moving target indication (MTI) performance; height finding without the need for an expensive 3-D radar; a relatively simple form of limited target recognition; and greater immunity to certain forms of electronic countermeasures (ECM).

The Senrad radar described here was developed as an R\&D experimental test bed to demonstrate new generic radar concepts. For this reason, it had characteristics that differed from those of an operational system. This was done for experimental convenience, but it does not significantly affect the demonstration of Senrad's basic capabilities.

\section{DESCRIPTION OF SENRAD EXPERIMENTAL RADAR}

The chief differences between Senrad and conventional 2-D air-surveillance radars were the very wide band over which it operated and the signal processing and algorithms it used to extract the benefits of wideband operation.

Choice of Frequency: The radar frequency band over which a radar operates influences greatly its capabilities and character. Table I lists the frequency bands which have generally been used for long-range air-surveillance radars. At some time during the history of radar, there have been operational air-surveillance radars in each of these various bands. The lower frequencies (VHF and UHF) offer the advantages of lower cost, freedom from weather effects, better MTI performance, and a slightly greater radar cross section of the target. The lower frequencies (such as VHF), however, suffer from having to operate in a crowded part of 
TABLE I

Frequency Bands That Have Been Used in the Past for Long-Range Air-Surveillance Radar

\begin{tabular}{cc}
\hline \hline Radar Band & Frequency $(\mathrm{MHz})$ \\
\hline VHF & $216-225$ \\
UHF & $420-450$ \\
UHF or L $\mathrm{u}_{\mathrm{u}}$ & $850-942$ \\
$\mathrm{~L}$ & $1215-1400$ \\
$\mathrm{~S}$ & $2700-2900$ \\
& $2900-3100$ \\
& $3100-3700$ \\
\hline
\end{tabular}

the electromagnetic spectrum, they have narrow bandwidths and broad beamwidths, and poor low-altitude coverage. At the higher frequencies (such as $\mathrm{S}$ band), there is more room in the spectrum for wideband operation, and resolution in the angle and range dimensions usually can be better than at the lower frequencies. Radars at the higher frequencies, on the other hand, generally cost more to achieve the same range performance as at lower frequencies; rain can seriously reduce target detection capability; and it is more difficult to achieve good MTI performance. Thus the middle microwave frequencies, such as $\mathrm{L}$ band, offer advantages not available at either higher or lower frequencies. ( $\mathrm{L}$ band is the unquestioned frequency band for long-range (enroute) civil air-traffic control radars as judged by the competition of the market place as well as being justified by analytical arguments.)

The International Telecommunications Union allocates for radar use the frequency range from 1215 to $1400 \mathrm{MHz}$, which is known as L band. There are many current, as well as previous, military and civil long-range air-surveillance radars within this band. There is also a neighboring radar band, from 850 to $942 \mathrm{MHz}$. According to the IEEE Letter-Band Standards for Radar [8], it is officially in the UHF region of the electromagnetic spectrum, but the technology and characteristics of radars in this frequency band are more like those at L band. For this reason, the upper part of the UHF band sometimes has been called $L_{u}$.

In considering the choice of frequency for the Senrad air-surveillance radar, we wanted to employ as much bandwidth as practical. Bandwidth is an important resource for radar since it represents information as well as allowing flexibility for radars. It was decided that the radar should operate simultaneously at both $\mathrm{L}$ and $\mathrm{L}_{\mathrm{u}}$ bands, covering from 850 to $1400 \mathrm{MHz}$. (Although the radar was designed to cover this entire band and could operate as an ultrawideband radar, there are other important nonradar users of these frequencies. Therefore the entire bandwidth was not available for radar use.) This is a relative bandwidth of $50 \%$, which is much greater than that of any other air-surveillance radar. In addition to operating in two bands with simultaneous frequency diversity, the radar is capable of changing its frequency on a pulse-to-pulse basis (frequency agility) when Doppler processing is not needed. When multiple pulses must all be at the same frequency in order to perform Doppler processing, frequency agility is on a waveform-to-waveform basis.

Benefits of Wideband Operation: The capabilities that are possible because of the use of multiple frequencies over an extremely wide bandwidth include the following.

1) Good automatic detection and track (ADT) because of the absence of fades (loss of signal). The deep interference nulls in the elevation coverage that result from surface-multipath reflection with a single narrowband signal are greatly reduced with the use of wideband frequency diversity. In addition to increasing the ability to detect targets, it makes ADT more reliable because there is no large loss of echo signal due to the target being in a deep null of the antenna multipath pattern.

2) Better MTI. Loss of echo signal because of MTI blind speeds is effectively eliminated by frequency diversity, without the need for multiple staggered pulse repetition frequencies.

3) Enhanced target cross section. The combined echo signals from a multiple-frequency radar is not likely to experience the very low values that can occur when a slowly fluctuating target is viewed with only a single frequency [9]. (This is similar to converting a Swerling case 1 cross section model to a Swerling case 2).

4) ECCM (electronic counter-countermeasures). Proper operation over a wide bandwidth forces a noise jammer to cover the entire band. Consequently, there is a reduction of the jammer power density the radar has to face compared with what it would be if all the jammer power were concentrated into a narrow bandwidth.

5) High range resolution. This allows height finding based on multipath, as well as providing a form of target recognition based on the target's range profile.

Block Diagram: The radar could have been built as one system with a single transmitter covering the entire $50 \%$ bandwidth. Instead, the use of two separate transmitters was preferred, one covering the lower portion of the total band and the other covering the upper portion. A block diagram of the experimental Senrad system is shown in Fig. 1. A diplexer connects the two radars to a single antenna. A single radar control computer and a single data processor are used. The characteristics of Senrad are summarized in Table II.

The use of two transmitters, rather than a single transmitter, to cover the $50 \%$ bandwidth offers a more technically feasible system with greater experimental 
Frequency: lower sub-band, 850 to $942 \mathrm{MHz}$ upper sub-band, 1215 to $1400 \mathrm{MHz}$

Antenna (parabolic reflector)

size: 24 by $6.5 \mathrm{ft}$

azimuth beamwidth: $3 \mathrm{deg}$ at $900 \mathrm{MHz} ; 2.3 \mathrm{deg}$ at $1300 \mathrm{MHz}$

azimuth peak sidelobe: $-22 \mathrm{~dB}$

elevation beam: cosecant-squared to $30 \mathrm{deg}$

gain: $27.2 \mathrm{~dB}$ at $900 \mathrm{MHz} ; 29.8 \mathrm{~dB}$ at $1300 \mathrm{MHz}$

polarization: horizontal

Antenna (low-sidelobe planar phased array)

size: 24 by $7.5 \mathrm{ft}$

azimuth beamwidth: $3.8 \mathrm{deg}$ at $900 \mathrm{MHz} ; 2.8 \mathrm{deg}$ at $1300 \mathrm{MHz}$

azimuth peak sidelobe: $-40 \mathrm{~dB}$

elevation beam: cosecant-squared to $30 \mathrm{deg}$

gain: $27.9 \mathrm{~dB}$ at $900 \mathrm{MHz} ; 29.0 \mathrm{~dB}$ at $1300 \mathrm{MHz}$

polarization: vertical

Data rate (revisit time): 4 or 8 seconds

Power (TWT): $160 \mathrm{~kW}$ peak power in each band; $6 \mathrm{~kW}$ average power in each band, but varies depending on the waveform used

Pulse widths: 10, 30, and $120 \mu \mathrm{s}$, compressed to $0.7 \mu \mathrm{s}$

Pulse repetition frequencies: waveform dependent

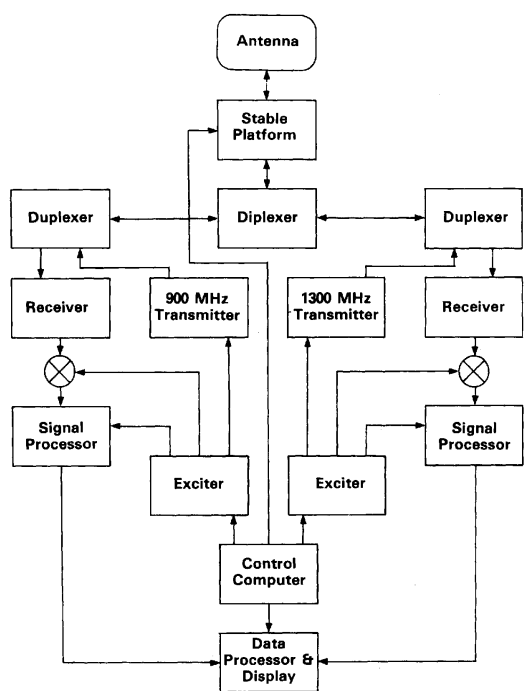

Fig. 1. Block diagram of the Senrad experimental radar with a single antenna and a lower-frequency sub-band and an upper-frequency sub-band.

flexibility and important operational advantages. Two transmitters allow simultaneous transmission at two widely separated frequencies, something not as practical with a single transmitter. A single wideband transmitter generally has to radiate multiple frequencies sequentially rather than simultaneously. Simultaneous operation was important in the Senrad concept since it makes the countermeasures threat easier to handle. Two separate radar transmitters (and receivers) also have the advantage of increased reliability since such a radar can perform as a conventional radar in case only one of the two sub-bands is operable.

It was also envisioned that for naval applications, a small ship needing a long-range air-surveillance radar might use a smaller version of the radar that operated in only one of the two sub-bands. Larger ships would employ both sub-bands while smaller ships might have either the upper or the lower sub-band. Using only one sub-band on smaller ships would still force jammers to cover the entire band since both sub-bands would likely be found within a naval task force.

Transmitter: The traveling wave tube (TWT) and the solid-state amplifier were considered suitable for this application since both are quite capable of the required bandwidth and both allow good MTI (detection of moving targets in clutter). Initially, it seemed possible to use a single TWT transmitter to cover the entire $50 \%$ band. This, however, would have required a special tube development, something usually too expensive for R\&D budgets. It was also considered that solid-state modules might be used to cover the entire band, either as a separate transmitter or with the modules located on a rotating array antenna-one module to a radiating element. This option might be more viable today, but it was not chosen at the time because of high cost; and it would require special development. Putting the solid-state transmitter on the antenna also increases the weight on the antenna, makes cooling more arduous, places more of a burden on maintenance, and makes it difficult to achieve very low sidelobe antennas. Thus, we chose to 


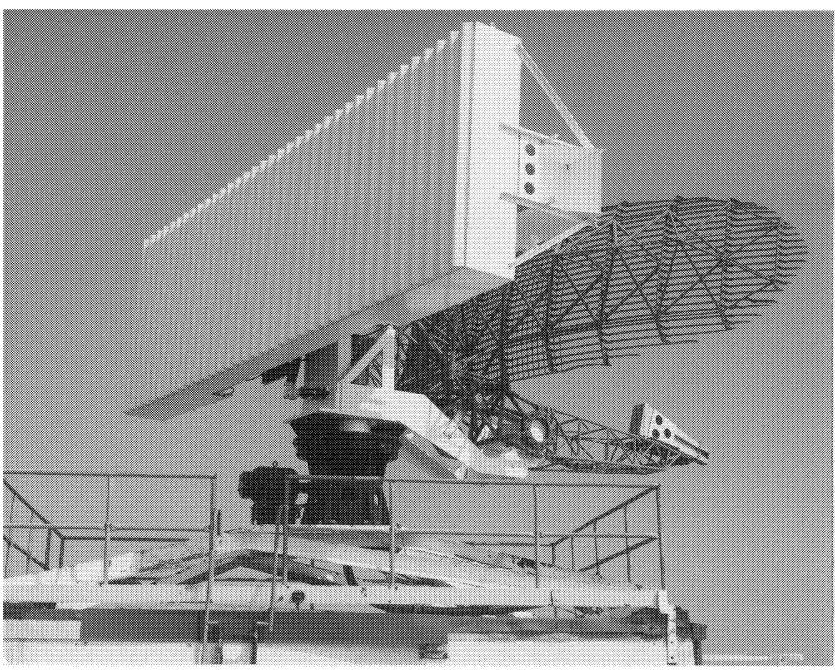

Fig. 2. Photo of mechanically rotating back-to-back experimental Senrad antennas; one is a conventional parabolic reflector, the other is a low-sidelobe planar array.

configure the transmitter with two TWTs: one to cover the lower frequencies $\left(\mathrm{L}_{\mathrm{u}}\right.$ band) and the other to cover the standard $\mathrm{L}$ band.

There was already a suitable operational TWT available (Raytheon QKW1671A) for the upper sub-band from 1215 to $1400 \mathrm{MHz}$, which was being used in other radar systems. It was a grid-pulsed, liquid-cooled, ring-bar TWT with an integral solenoid for focusing. The tube had a gain of $50 \mathrm{~dB}$, which permitted the use of a low-power solid-state driver. At the lower sub-band, a frequency-scaled version of the upper sub-band TWT was developed, and was known as the QKW1818. Test data showed that this tube was capable of operating from 850 to $1300 \mathrm{MHz}$. Both tubes had similar characteristics. Their peak power output was nominally $160 \mathrm{~kW}$ and their average power could be as high as $10 \mathrm{~kW}$. In the experimental Senrad system, the tubes were operated at about half of their average power so as to reduce cost and risk. The power supply for the experimental radar was a surplus dual-tube life-test station, modified for these purposes.

By using two transmitters, a separate duplexer could be employed at each sub-band. High-power duplexers to cover the entire band from 850 to 1400 $\mathrm{MHz}$ did not exist at the time, so a major and risky development to obtain a suitable duplexer would have had to be undertaken if a single wideband system were used. A broadband, high-power diplexer was designed to combine the outputs of the two transmitters to feed a single antenna.

Antenna: The experimental system utilized two different types of mechanically rotating antennas. One was a conventional parabolic reflector and the other was a low-sidelobe array antenna. A photograph of the two antennas on the roof of the Radar Division building at the NRL Chesapeake Bay Field Site is shown in Fig. 2. They are mounted back-to-back for experimental convenience. (Only one antenna would be used in an operational system.) The particular parabolic-reflector antenna employed in this experimental equipment was the antenna from the developmental model of the Navy's AN/SPS-50, an L-band cousin of the $\mathrm{L}_{\mathrm{u}}$-band AN/SPS-49. A broadband double-ridged waveguide feed covering the entire $850-1400 \mathrm{MHz}$ frequency band was designed and built. The antenna was $24 \mathrm{ft}$ wide by $6.5 \mathrm{ft}$ high and it radiated horizontal polarization. It had an azimuth beamwidth of $3^{\circ}$ at $900 \mathrm{MHz}$ and $2.3^{\circ}$ at $1300 \mathrm{MHz}$. The elevation coverage extended to $30 \mathrm{deg}$ with cosecant-squared shaping, but with $3 \mathrm{~dB}$ enhancement of the gain above an elevation angle of $20^{\circ}$. Its peak sidelobe was $-22 \mathrm{~dB}$ and gain was $27.2 \mathrm{~dB}$ at $900 \mathrm{MHz}$ and $29.8 \mathrm{~dB}$ at $1300 \mathrm{MHz}$.

The parabolic reflector was used for the early experiments, but later in the program a low-sidelobe antenna was developed for Senrad by Westinghouse (now Northrop Grumman). It consisted of 16 rows of 36 dipole radiators each, and was 24 by $7.5 \mathrm{ft}$ with an azimuth beamwidth from 3.8 to $2.8^{\circ}$ depending on frequency. The elevation coverage was cosecant-squared up to $30^{\circ}$. The gain was nominally $28.5 \mathrm{~dB}$. Its peak sidelobe was designed to be $-40 \mathrm{~dB}$. This sidelobe level might not seem "bold," but it should be remembered that the antenna was of relatively low gain compared with other low-sidelobe antennas. The lower the gain the more difficult it is to reduce the sidelobes to low levels. The vertical polarization of the low-sidelobe antenna helped in making comparisons of the effect of polarization with respect to the horizontally polarized reflector antenna.

The antenna rotation rate was $15 \mathrm{rpm}$ ( $4 \mathrm{~s}$ revisit time), which is much faster than the 5 or $6 \mathrm{rpm}$ common with long-range civil air-traffic control radars.

\section{WAVEFORMS}

Modern air-surveillance radars often employ more than one waveform to obtain satisfactory performance under a variety of environmental and operational conditions. In Senrad, there were three basic surveillance waveforms: 1) long-range, 2) clear-sky MTI, and 3) rain and chaff MTI. (There were many other choices of waveforms that could be readily selected by keyboard entry.) These waveforms of the experimental Senrad used pulsewidths from 10 to $120 \mu \mathrm{s}$, each with $2 \mathrm{MHz}$ bandwidth linear-FM pulse compression that produced a $0.7 \mu$ s compressed pulsewidth with $-30 \mathrm{~dB}$ time sidelobes. To reduce the effectiveness of hostile electronic warfare, there were simultaneous transmissions at different frequencies in each of the two Senrad sub-bands.

Long-Range Surveillance Waveform: In the experimental system this was a single high-power 


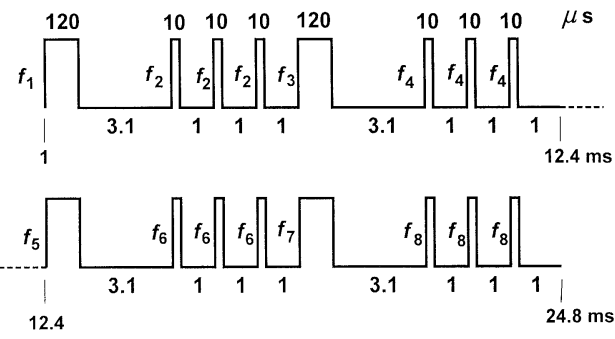

Fig. 3. Clear-sky waveform showing the long-range surveillance pulses $(120 \mu \mathrm{s})$ at frequencies $f_{1}, f_{3}, f_{5}, f_{7}$, and the three pulses $(10 \mu \mathrm{s})$ of the MTI waveform at frequencies $f_{2}, f_{4}, f_{6}, f_{8}$. Time between the MTI pulses was $1 \mathrm{~ms}$ and the time between a longrange pulse and the start of the 3-pulse MTI waveform was $3.1 \mathrm{~ms}$.

$120 \mu$ s pulse whose function was to provide detection at long range in the absence of clutter. (In an operational system the pulsewidth would be $250 \mu \mathrm{s}$.) The instrumented range was $250 \mathrm{nmi}$. The minimum time on target of $25.6 \mathrm{~ms}$ at the highest frequency (1400 MHz with the $2.3^{\circ}$ reflector antenna and a $4 \mathrm{~s}$ revisit time) results in at least four such long-range surveillance pulses transmitted in both sub-bands, Fig. 3. Each of these transmitted pulses can be, and usually was, at a different frequency during the time on target.

Clear-Sky MTI Waveform: To detect aircraft in surface clutter, a three-pulse MTI waveform was interspersed with the long-range surveillance pulses, as in Fig. 3. The $10 \mu$ s pulses of the MTI waveform were separated by $1 \mathrm{~ms}$. MTI processing was implemented out to a range of $80 \mathrm{nmi}$. The time between the long-range pulses was $6.1 \mathrm{~ms}$.

Fig. 3 illustrates the long-range surveillance pulses interspersed with the 3-pulse MTI waveforms for one of the two sub-bands. With a $15 \mathrm{rpm}$ antenna rotation rate, the time taken for the half-power points of the parabolic reflector antenna beam to scan by a point target was approximately $25.6 \mathrm{~ms}$ at the highest frequency of almost $1400 \mathrm{MHz}$, and $33.3 \mathrm{~ms}$ at the lowest frequency of $850 \mathrm{MHz}$. The duration of the waveform shown in Fig. 3 was 24.8 ms. Thus the complete sequence of pulses shown in this figure was able to illuminate a target even at the highest radar frequency where the antenna beamwidth was smallest. As a minimum, therefore, each target was illuminated by four long-range surveillance waveforms and four MTI waveforms in each sub-band, and each could be at a different frequency. The three MTI pulses, however, had to be at the same frequency for doppler processing to be obtained. This is unavoidable and can make the radar more vulnerable to ECM. Senrad, therefore, could operate with up to eight different frequencies radiated within the higher frequency sub-band during the time on target, and more than eight at the lower sub-band. Operation of a radar over a wide frequency range forces a jammer to dilute its radiated power-density by spreading the total energy over a very wide band.
Rain and Chaff Waveform: In the presence of wind-blown rain or chaff, doppler processing must be able to filter the moving volumetric clutter as well as the stationary surface clutter. A sequence of 14 pulses was originally considered for this waveform with processing being performed by two three-pulse cancelers in cascade. One three-pulse canceler had its doppler rejection notch centered on the velocity of the surface clutter (sea or land). The other three-pulse canceler had its doppler rejection notch centered on the average velocity of the volumetric clutter (rain or chaff). This was replaced by a ten-pulse waveform with $30 \mu$ s pulsewidths to eliminate both surface and volume clutter similar to that which is done in the Moving Target Detector [10]. A three-pulse canceler with binomial weighting was followed by an eight-pulse doppler filter bank which used an eight-point fast Fourier transform (FFT) with a log-CFAR (constant false alarm rate) normalizer [11] at the output of each filter. The sidelobes of the doppler filter bank were $-29 \mathrm{~dB}$. The basic unambiguous range of this waveform was approximately $80 \mathrm{nmi}$, so that a range ambiguity could occur for long-range targets. A shift in the PRF between the first ten-pulse group and the second ten-pulse group permitted resolution of the range ambiguity between the first and second $80 \mathrm{nmi}$ intervals. Alternate groups of ten pulses were transmitted on different frequencies. As with the clear-sky waveform, simultaneous transmissions were made in both sub-bands so that the rain and chaff waveform radiated on at least four frequencies during the time on target.

Range-extent gates (which look for extended echoes in more than two adjacent range resolution cells) were used in conjunction with the FFT doppler filter-bank to eliminate range-extended land, sea, or rain clutter, as well as chaff echoes. A blanker loop was closed around the doppler processor to remove low-velocity clutter echoes and large fixed-clutter residues which were not sufficiently extended in range to be removed by the range-extent gates and were sufficiently large so as to exceed the attenuation of the first three-pulse MTI canceler.

Pulse-to-Pulse Frequency Agility: The clear-sky and the rain and chaff waveforms both employed a form of frequency agility, but not pulse-to-pulse agility. The long-range waveform described above, however, is an example of a pulse-to-pulse frequency-agile waveform. When there is no clutter to degrade the detectability of targets, Senrad can transmit a pulse-to-pulse frequency agile waveform covering a very wide band of frequencies to reduce the harmful effects of hostile jamming.

Other Waveforms: A wideband Stretch [12] waveform was used for both target recognition and multipath height finding. It could have any time duration and bandwidth that was supportable by the 


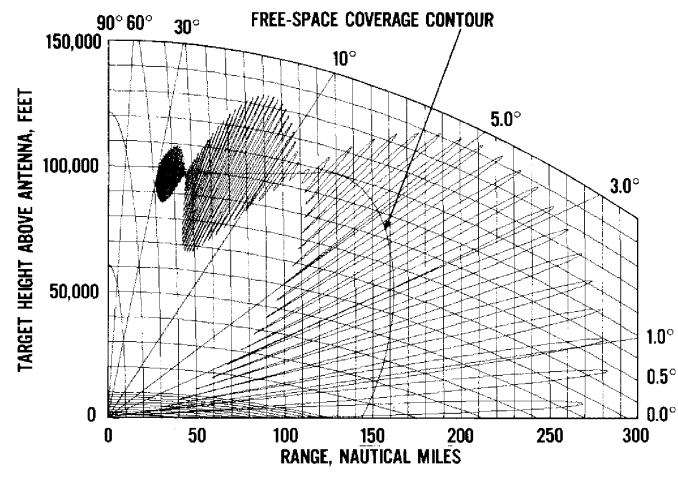

(a)

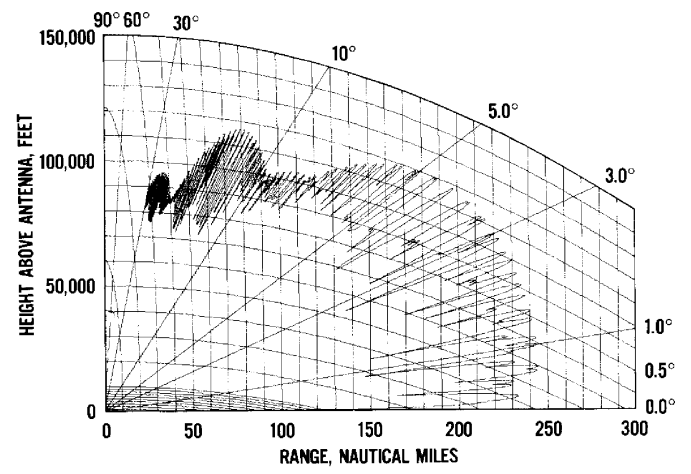

(b)

Fig. 4. Calculated Senrad coverage in elevation. (a) Single-frequency coverage. (b) Coverage with four different frequencies within the Senrad frequency band.

transmitter, but was often used with a $100 \mu$ s pulse duration. Considerable flexibility was built into the experimental system so that radar parameters could be easily changed by computer control.

\section{PERFORMANCE}

In this section, examples are given of the predicted and demonstrated performance obtained by the Senrad radar system.

Continuous Coverage: The calculated elevation coverage based on parameters of a larger version of the experimental system is shown in Fig. 4(a) at a single frequency of $900 \mathrm{MHz}$ and a target cross section of $1 \mathrm{~m}^{2}$. The usual lobed elevation pattern is seen with its deep nulls. A radar observing an aircraft flying at a constant altitude would lose the echo periodically as the target flew in the null regions between the lobes. Consequently, tracking will likely be poor or even dropped when the target is located within the antenna nulls. If dropping of a track occurs, the track can be reinitiated when the target appears in another lobe; but reinitiating a track takes time and computer resources. The composite coverage obtained with Senrad when it used four frequencies $(850,940$, 1215, and $1400 \mathrm{MHz}$ ) is shown in Fig. 4(b). The nulls in the pattern are fairly well filled in, so that tracking is almost continuous.

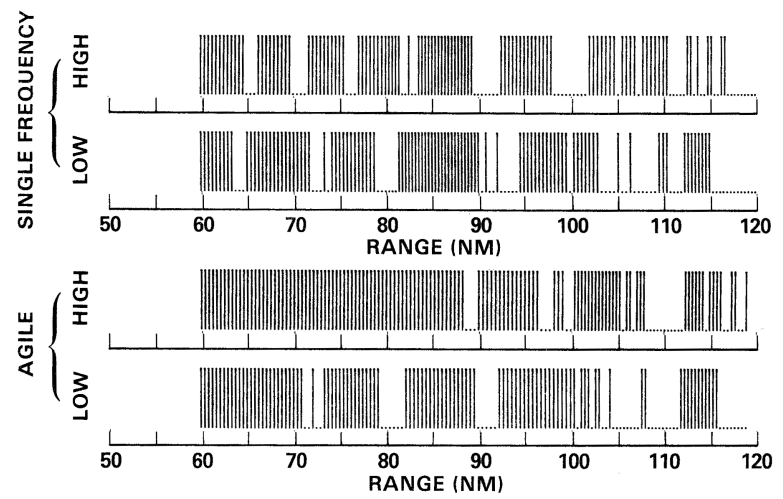

Fig. 5. Detection performance (blip-scan ratio) of experimental Senrad with $30 \mu$ s pulse with a single frequency (top) and with four frequencies (bottom).

Fig. 5 is an example of the measured detection capability of the experimental Senrad system obtained with a $30 \mu$ s pulsewidth over the range from 60 to $120 \mathrm{nmi}$ when viewing an S2D aircraft (a two-engine propeller-driven ASW aircraft small enough to fly off an aircraft carrier.) The blip-scan ratio ${ }^{1}$ was found to be 0.78 in either the upper or lower sub-bands when only a single frequency was transmitted. On the other hand when multiple frequencies were used in both sub-bands, the blip-scan ratio became 0.98 .

Fig. 6 is another experimental demonstration of how multiple frequencies can improve detection. The range rings in these two offset plan-position indicator (PPI) displays are spaced $50 \mathrm{nmi}$ apart. The shutter of the scope camera remained open for 8 scans of the antenna. The display in Fig. 6(a) is with a single frequency. None of the targets can be seen on all eight scans of the antenna. The display in Fig. 6(b) is with multiple Senrad frequencies. All targets are seen on all eight scans. (Note that these two displays were not taken at the same time.)

ECCM: The main ECCM feature of Senrad that was examined was its frequency agility and frequency diversity over a $50 \%$ bandwidth. Fig. 7 shows the reduction of jamming if the jammer could be forced to spread its available energy over a wide bandwidth. In the PPI display of Fig. 7(a) the jammer was allowed to concentrate its available power over a signal bandwidth of $30 \mathrm{MHz}$. The conventional reflector antenna was used in this case. Only a few targets are seen since the radar utilized CFAR which raises the receiver threshold when external noise increases. In Fig. 7(b) the low-sidelobe antenna is used and the jammer power is spread over a much larger bandwidth of $400 \mathrm{MHz}$. The number of targets seen is much greater.

\footnotetext{
${ }^{1}$ The blip-scan ratio is an experimentally measured approximation to the single-scan probability of detection of an aircraft. It is defined as the ratio of the number of scans on which the aircraft target was seen to the number of times it could have been seen. The aircraft usually flies back and forth at constant altitude on a radial path.
} 


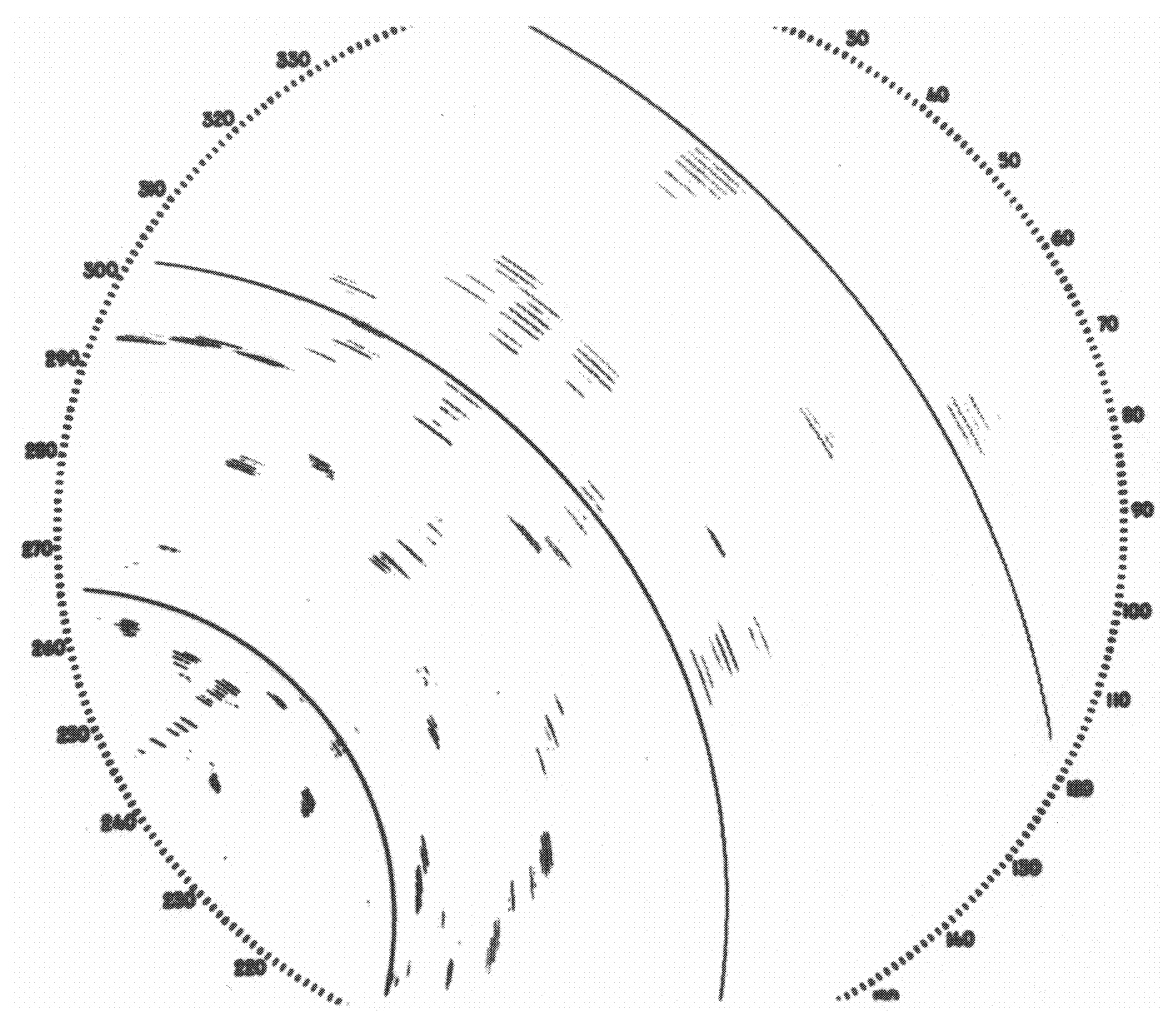

(a)

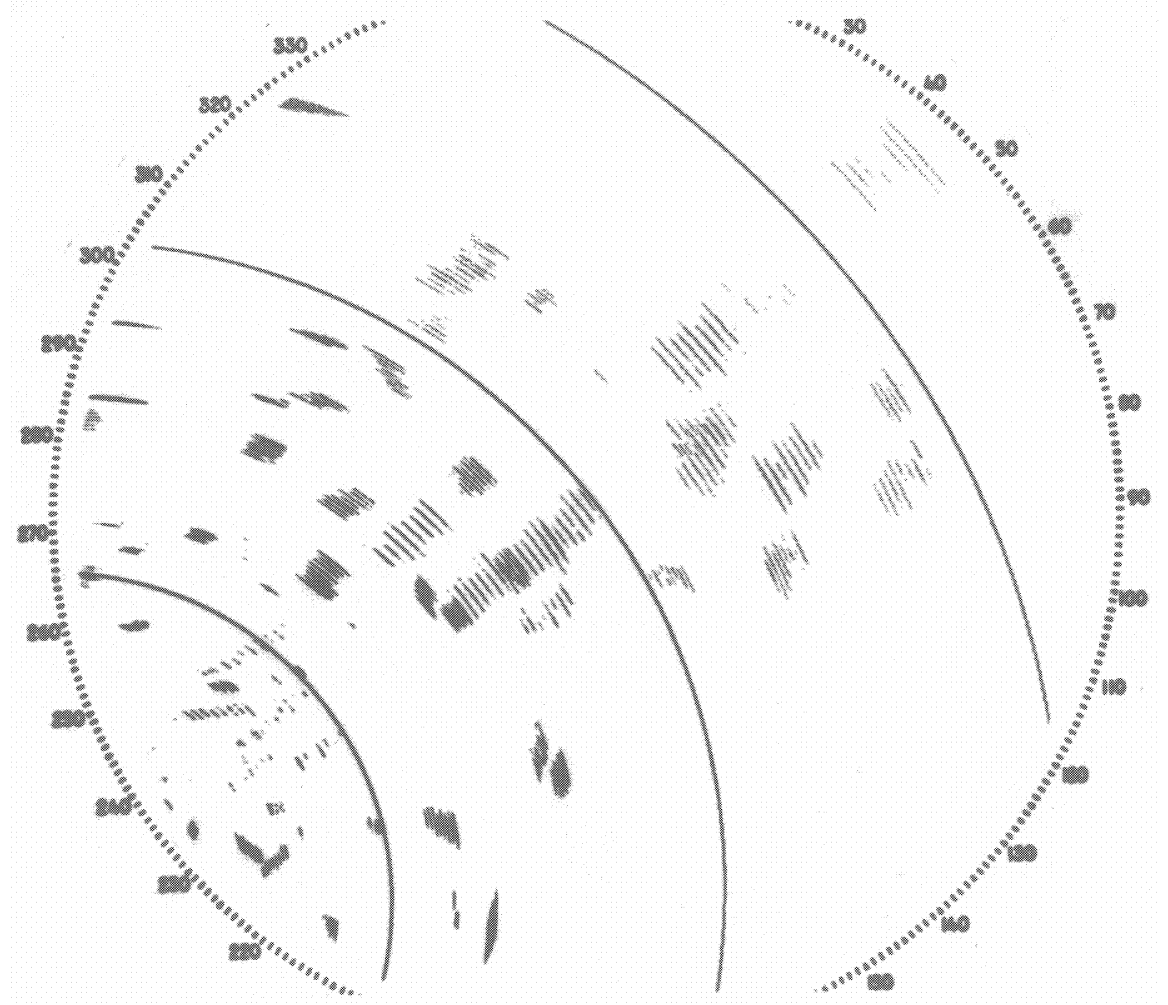

(b)

Fig. 6. Offset PPI display of experimental Senrad system showing eight scans of the radar. (a) With single frequency. (b) With four frequencies. 


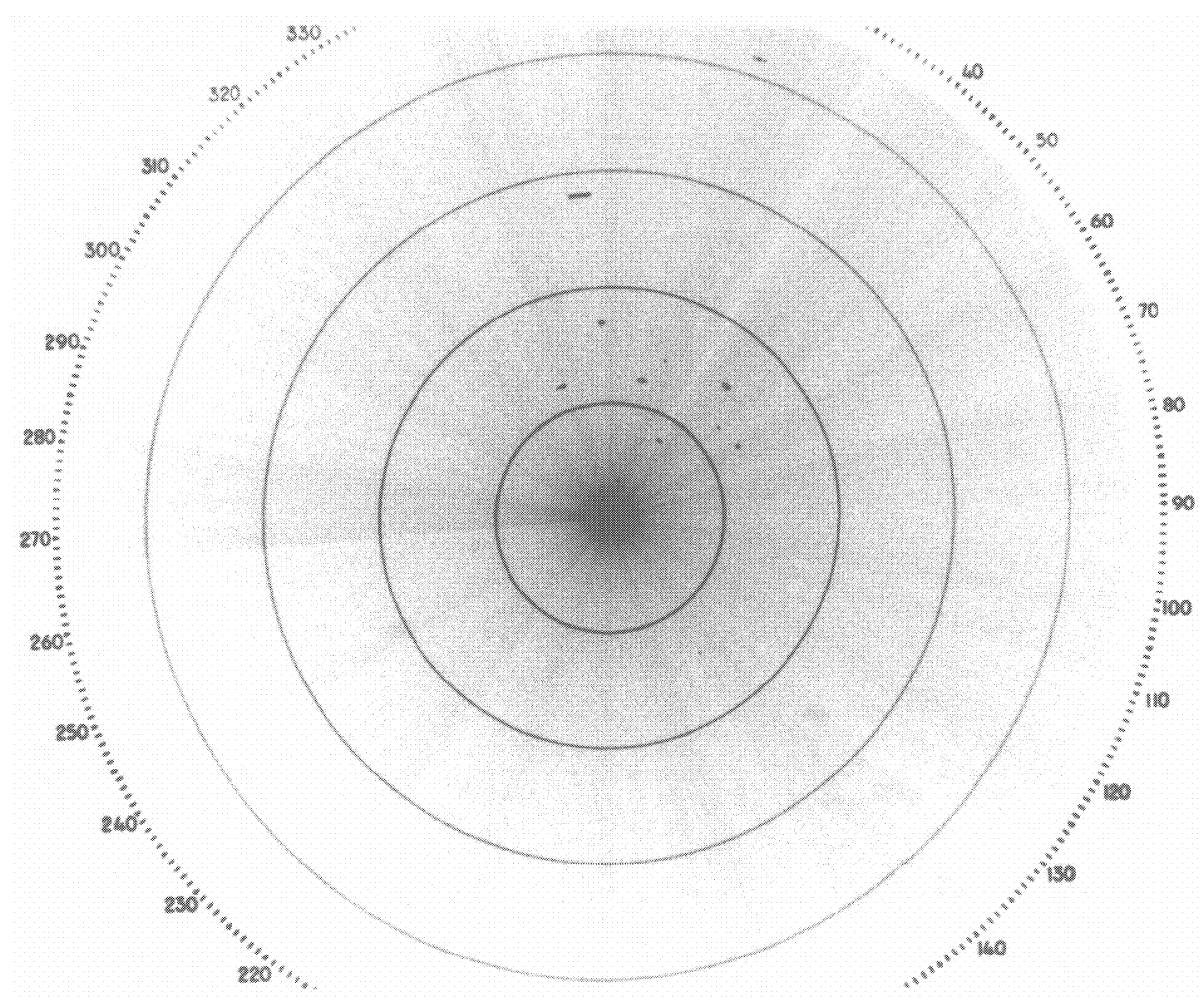

(a)

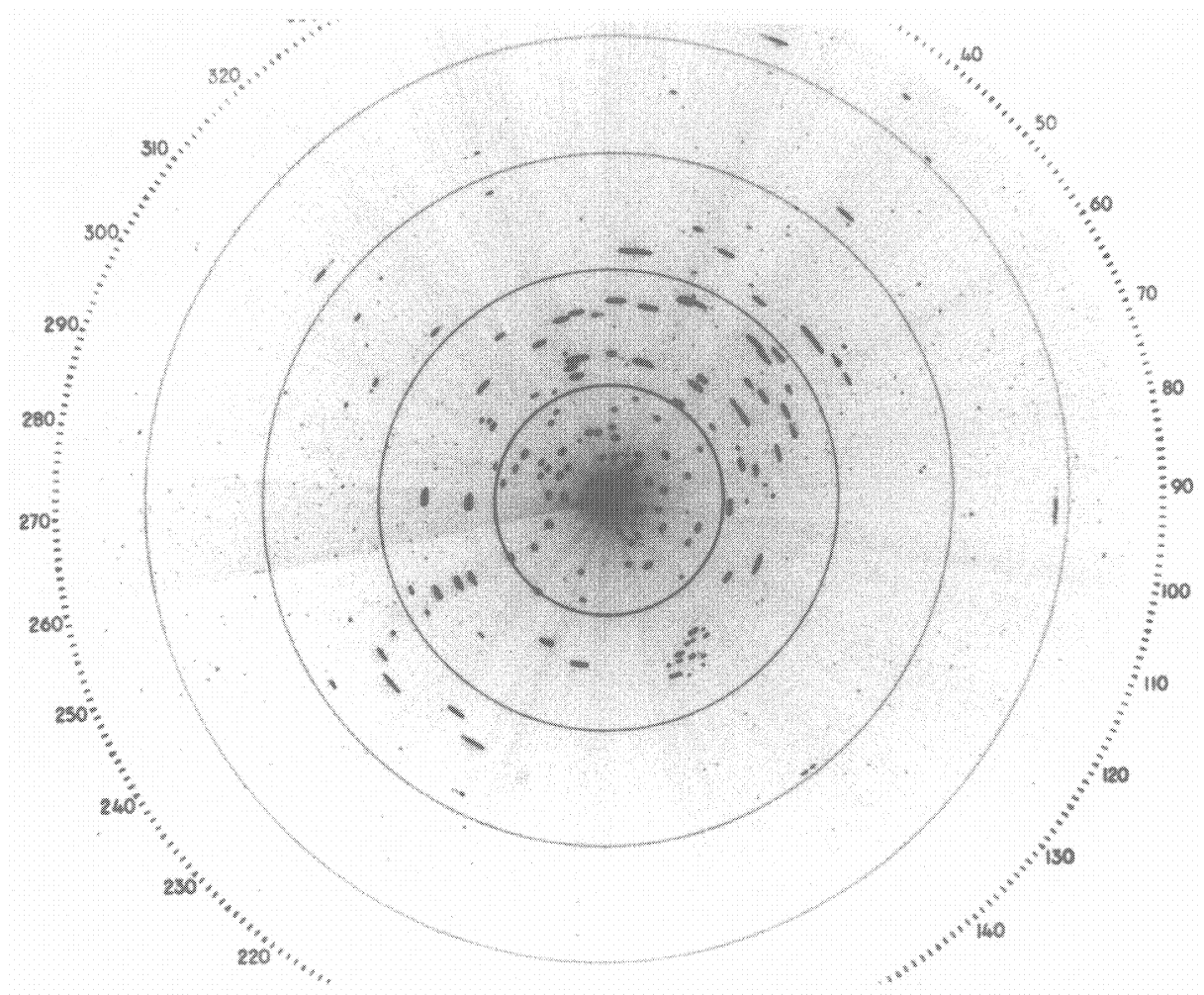

(b)

Fig. 7. Effect of bandwidth and antenna sidelobes on barrage jamming. (a) Jammer concentrates its power within $30 \mathrm{MHz}$, with conventional Senrad antenna. (b) Jammer spreads its power over $400 \mathrm{MHz}$, with low-sidelobe Senrad antenna. (Since the radar used CFAR, the more effective the jammer the fewer the targets that are seen.) 


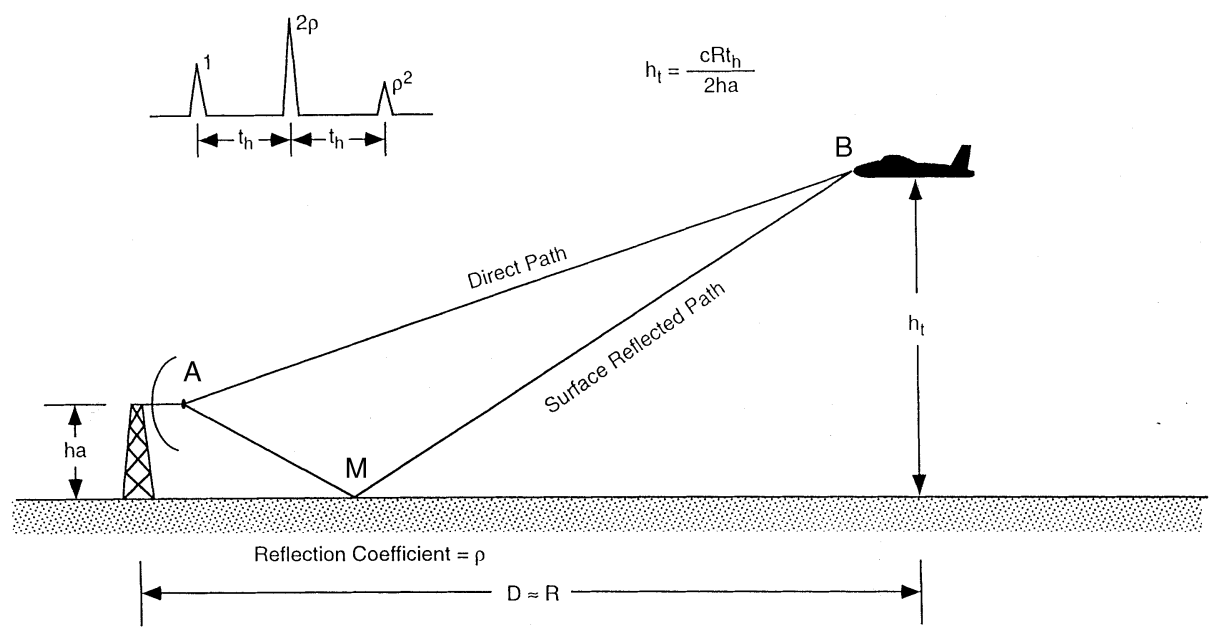

Fig. 8. Multipath for determining target height.

In addition to operating over a wide bandwidth, a good military radar would incorporate many other ECCM techniques such as sidelobe cancellation, prelook, recognition of repeater jamming, and others [13].

High-Resolution Operation: Conventional air-surveillance radars typically have pulsewidths of one or a few microseconds, which result in range resolutions of several hundreds of meters. This is usually satisfactory for accomplishing most air-surveillance-radar tasks. The wide bandwidth of Senrad in both the upper and lower sub-bands, however, offers capabilities not available with the narrow bandwidths typical of conventional surveillance radars. The use of wideband radar waveforms for high range-resolution is well known as a method for reducing the amount of distributed rain-clutter and surface-clutter echoes with which the target echo has to compete. If there is sufficient resolution to separate the direct target-echo from the surface-reflected target-echo, Fig. 8, there will be no fading of the target echo signals due to multipath nulls in the elevation antenna pattern. Both of the above capabilities (clutter reduction and separation of multipath) can enhance detection. A high-resolution waveform in Senrad was not used for increasing the probability of detection in clutter during surveillance, but to gather additional information on targets that had already been detected. In particular, the wide bandwidth was used to obtain 1) target height by measuring the time delay between multipath echoes, and 2) by performing a degree of limited target recognition (sometimes called perceptual class recognition) based on the target's profile in the range coordinate. Both of these tasks were performed with Senrad using a linear-FM Stretch pulse-compression waveform.

When high-resolution waveforms were used, they were only radiated as needed. For example, as the radar scans by a target, detection can often occur during the first few pulses transmitted. If either height finding or target recognition were required on a target, one or more of the pulses within the dwell time (or time-on-target) would be modulated with a wide bandwidth high-resolution Stretch pulse-compression waveform to obtain target height and/or recognition.

Height Finding: The height of a target traditionally is found with a 3-D air-surveillance radar; one that utilizes multiple beams in elevation or employs one or more scanning pencil-beams to find the elevation angle of the target [14]. The 3-D radar has been widely used in the past even though it might have been from four to ten times the cost of a 2-D (range and azimuth) air-surveillance radar. A 3-D radar generally has poor height accuracy at low angles because of multipath effects when the main beam of the antenna illuminates the land or sea surface. A 3-D antenna was examined for Senrad, but not implemented. We instead chose to explore the use of multipath for obtaining the third target coordinate, which is height.

Finding target height based on the multipath echo structure in a 2-D wide-bandwidth radar has some important advantages compared with finding height using conventional 3-D radars. With sufficient range resolution, the direct signal can be separated from the surface-reflected signal. The time separation between these signals depends on the target height, Fig. 8. A flat-Earth is assumed. There are four components to the multipath echo $[15,16]$. The first is the direct path $\mathrm{AB}$ and return over the same path. The second is the path $\mathrm{AMB}$ and return via $\mathrm{AB}$, or $\mathrm{AMB}+\mathrm{AB}$. There is also the opposite path of the same length (time delay); that is, $\mathrm{AB}+\mathrm{AMB}$. The fourth path is the path $\mathrm{AMB}$ out and back. The time $t_{h}$ in Fig. 8 can be found as [17]

$$
t_{h}=2 h_{a} h_{t} / c R
$$

where $h_{a}=$ radar antenna height, $h_{t}=$ target height, $c=$ velocity of propagation, and $R=$ range. If we note 
that $h_{t} / R$ is the elevation angle $\theta_{e}$ to the target, then

$$
\theta_{e}=c t_{h} / 2 h_{a} \text {. }
$$

For example, if $h_{a}=100 \mathrm{ft}, h_{t}=10,000 \mathrm{ft}$, and $R=50 \mathrm{nmi}$, the time $t_{h}$ is $6.69 \mathrm{~ns}$, which requires a bandwidth of at least $149.5 \mathrm{MHz}$. This bandwidth is well within the capability of a radar like Senrad. In practice the experimental Senrad system with Stretch pulse compression had a range resolution of $1 \mathrm{~m}$ and its antenna height was $55 \mathrm{~m}$ above the surface. If it is assumed that the multipath height-finding method is applicable when the pulsewidth $\tau<t_{h}$, then from (2) it is found that the experimental Senrad could utilize the multipath height finding method so long as the target's elevation angle was greater than about $1 \mathrm{deg}$.

The above applies for a flat Earth, and has to be modified for a spherical Earth. In most cases of interest for a surface-based air-surveillance radar, such as considered here, the antenna height is small and the target height is much larger so that the reflection from the Earth can be approximated using the flat-Earth assumption. This gives the height above the tangent plane at the radar, but the altitude above the Earth's surface can then be found from a radar range-height-angle chart [18]. This is the method that was used to find the height with Senrad [19]. When a flat-Earth approximation and a range-height-angle chart cannot be used, as when both an airborne radar and the target are at a high altitude above the Earth's surface, then the relation between the measured time delay and the target altitude has to be determined using a more sophisticated model taking account of the height of the radar above a round Earth.

In most cases of practical interest with multipath height-finding using a surface-based radar, the temporal extent of the echo received from an aircraft target can be much greater than the multipath time delay $t_{h}$ so there is overlapping of the three multipath echoes. When this occurs, as it did in Senrad, finding the time delay based on a time-domain measurement is difficult. Instead, the time delay can be found by taking the autocorrelation of the echo signal. The first peak of the autocorrelation function is usually the time corresponding to $t_{h}$. The use of the autocorrelation function requires that there be significant multipath effect (that is, the surface reflection coefficient should be large), and the target must have several resolvable scattering centers. The use of the autocorrelation function was how the time delay $t_{h}$ was found with Senrad.

The high-resolution waveform used for multipath height-finding was a linear-FM Stretch pulse compression waveform with a range resolution of about $1 \mathrm{~m}$. As the antenna beam scanned past the target a detection was made in a conventional manner and, before the beam left the target, a high-resolution waveform was substituted for one of the long-range detection pulses. The experimentally measured elevation-angle accuracy derived from the multipath echo was quite good. When the altitude found by the radar was compared with the barometric altitude reported by the aircraft's ATCRBS (Air Traffic Control Radar Beacon System) transponder, the elevation-angle error was approximately $0.13 \mathrm{deg}$ when the target was at an elevation angle of $1 \mathrm{deg}$ and $0.05 \mathrm{deg}$ at $5 \mathrm{deg}$ elevation. (The higher the elevation angle to the target, the greater will be the separation of the multipath echoes and the easier it is to measure an accurate time separation $t_{h}$.) These accuracies are difficult to achieve by other radar methods when the elevation beam is wide enough to illuminate the surface of the Earth to cause multipath.

Height-finding by means of conventional 3-D elevation-angle measurement methods can be highly inaccurate when the target is at low angles and the main beam of the antenna illuminates the surface [14] On the other hand, multipath height-finding works well at low angles if there is sufficient bandwidth. The multipath measurement is less satisfactory when the elevation angles are large and the surface-reflected multipath signal experiences a smaller reflection coefficient. When there is no multipath, however, the angle measurement using a conventional height finding method such as monopulse is not degraded so that the two methods, monopulse height-finding and multipath height-finding, are thus complementary.

[It was suggested by someone who reviewed the manuscript of this paper that if the range resolution were high enough to resolve the three multipath components shown in Fig. 8, then a monopulse elevation-angle measurement (and the target height) can be obtained by resolving the first (direct path) echo. This is correct for a point target or one whose radial echo extent in space is small compared with $c t_{h} / 2$. This does not usually happen for a surface-based radar such as Senrad where the extent of the target echo is greater than the time $t_{h}$. Finding the autocorrelation function of the entire echo signal when there are three overlapping distributed components takes advantage of all the echo components, and not just the leading edge of the direct path echo. The autocorrelation method that was used seems to be a simpler, more accurate, and less expensive method than a wide bandwidth, high range-resolution monopulse.]

Noncooperative Target Recognition (NCTR) [20, 21]: The high range-resolution offered by Senrad can be used to provide a form of limited NCTR based on the nature of the radar echo. The class of an aircraft target (that is, whether it is an F-15 or an F-18) is not easy to achieve in practical situations from the range profile alone $[22,23]$. Such a capability requires highly precise knowledge of the target aspect along with knowledge of the true target range-profile as a function of aspect. The range profile, however, can provide what has been called 

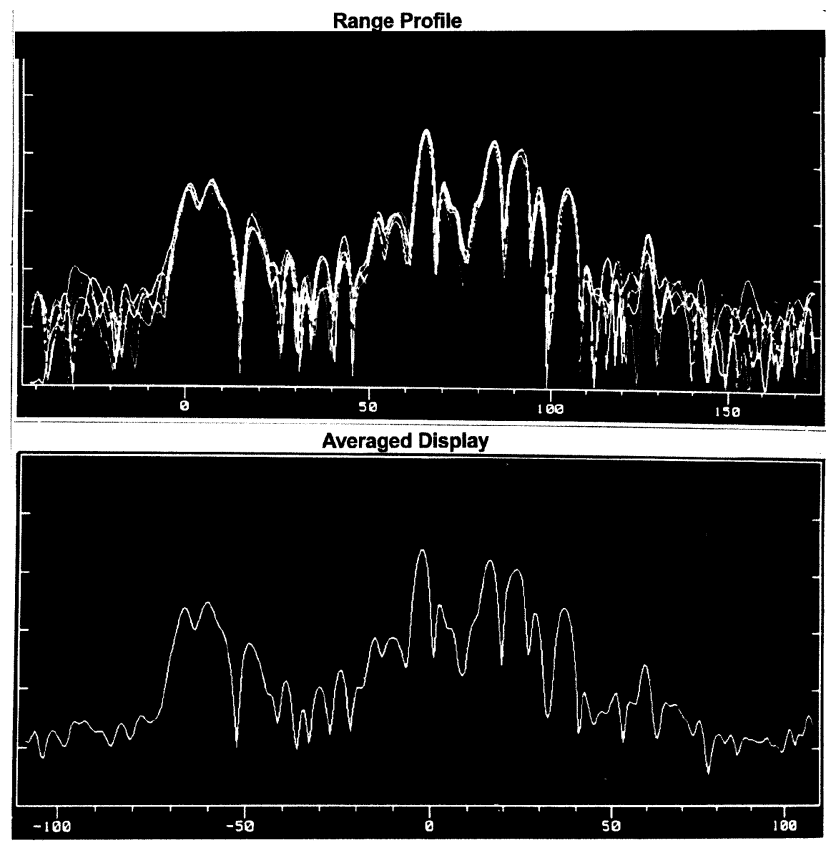

Fig. 9. In top portion of the figure is the superposition of seven pulse-to-pulse high range-resolution profiles of a Boeing 757 aircraft. After correction for the range change between observations, the aligned seven profiles are shown in the lower part of the figure.

perceptual classification. Perceptual classification means that aircraft targets can be sorted into the following rudimentary types: large jet, small jet, large propeller aircraft, small propeller aircraft, helicopter, missile, and decoy. In addition to using the high-resolution radial profile, recognition of perceptual class can also be assisted by estimating the radar cross section of the target, recognizing propeller modulation and its relative location, and sometimes the target speed. (By resolving the individual scatterers of a distributed target, a more stable estimate of the radar cross section can be obtained than when a long pulse is used.)

In the upper portion of Fig. 9 is the superposition of seven pulse-to-pulse radial profiles of a jet aircraft when the range resolution is about $1 \mathrm{~m}$. Knowledge of the target's radial velocity is needed to align in time the several range profiles. The high range-resolution of this waveform allows the radial velocity to be estimated by measuring the change of range that occurs between the first and the last pulse within a single dwell. This can provide an accurate estimate of radial velocity without the ambiguities that occur when radial velocity is found from the doppler frequency shift. Using the measured rate of change of range to time-align the seven radial profiles produces the average profile shown in the lower part of Fig. 9. From the target extent obtained from the averaged profiles and the aspect angle, a target length or wing profile can be estimated. This aircraft, a Boeing 757, was correctly classified as a "large jet." Propeller-driven aircraft are recognized by the echoes from the nose and tail areas being relatively constant, and the echoes from propellers having large fluctuations in amplitude.

In the experiments on target recognition, only the upper sub-band of Senrad was used.

Communications Via Radar: Although it was not a direct result of the wide bandwidth available in Senrad, the radar was also used as a test bed to demonstrate the advantages of using a radar to communicate to nearby radars. Communication between netted radars that observe the same coverage offers advantages for more reliable detection and more accurate tracking. This can occur within the radars of a naval task force or the air-defense radars of large ground forces. Conventional military communications links for command and control can be used to transfer radar data among radars, but radar data might not always have sufficient priority over such links to insure timely transfer of target information from one radar to another. Using the radar itself as the communications transmitter, however, allows the rapid transfer of information among the netted radars. Furthermore, high-power and high transmitting antenna gain make this communications link much less vulnerable to hostile jamming.

Only processed radar data, not the raw radar output, is communicated. Processed data is of relatively low information bandwidth compared with raw radar data. Since a radar pulse generally supports much greater bandwidth than the bandwidth required to transmit processed data, only a small portion of the radar's signal bandwidth need be used for transmitting radar information. The receiver at the other end of the communications link is a separate omni-directional antenna, permitting information to be communicated effectively whenever the radar antenna illuminates a nearby radar. For naval applications, the radars to be netted by communications generally will be within the line of sight of one another. The high power of the transmitted signal, however, will likely allow some propagation beyond the geometric line of sight.

ATCRBS and IFF: Since the Mark XII IFF (Identification Friend or Foe) cooperative aircraft identification system and the FAA's ATCRBS were within the frequency capability of Senrad, the low sub-band transmitter was used to generate interrogation waveforms for cooperative aircraft identification. The received signal was filtered and processed by a conventional IFF decoder. Very long-range interrogations and responses were achieved with the large antenna and high power offered by Senrad.

\section{CLOSING COMMENTS}

The Senrad experimental air-surveillance radar was operated for many years at the NRL Chesapeake Bay Field Site to demonstrate the significant benefits of very wideband frequency agility and frequency 
diversity. The ability to operate over a wide bandwidth in this manner improved detection and tracking, as well as lessened the threat of countermeasures and provided capabilities for finding target height without the need for an expensive 3-D radar. Senrad cannot recognize one class of aircraft from another, but it can be used to determine the general type of aircraft target. The technology for achieving these capabilities has been demonstrated, as well as the ability to operate within a wide bandwidth without serious interference to other users. This investigation has established the feasibility and the utility of the Senrad concept for a very wideband radar.

\section{ACKNOWLEDGMENTS}

The work reported here was performed by members of the Search Radar Branch of the Radar Division of the Naval Research Laboratory. Those who made major contributions to the success of this radar development and its evaluation include Dr. Robert Adams (former head of the NRL Search Radar Branch), William A. Douglas, W. Harold Harper, William L. Thrift, Raymond P. Meixner, T. P. Crowfoot, Owen F. Borchardt, and Robert Tompkins.

\section{REFERENCES}

[1] Herb, R. G., and Sinsheimer, R. L. (1947)

Examples of radar system design.

In L. N. Ridenour (Ed.), Radar System Engineering.

New York: McGraw-Hill, 1947, ch. 15.

[2] Shrader, W. W. (1973)

Radar technology applied to air traffic control. IEEE Transactions on Communication, COM-21 (May 1973), 591-605.

[3] Giaccaci, E., and Nucci, G. (1979) A family of air traffic control radars. IEEE Transactions on Aerospace and Electronic Systems, AES-15 (May 1979), 378-396.

[4] Taylor, J. W., Jr., and Brunins, G. (1985)

Design of a new airport surveillance radar (ASR-9).

Proceedings of the IEEE, 73 (Feb. 1985), 284-289.

[5] Skolnik, M. I. (1985)

Fifty years of radar.

Proceedings of the IEEE, 73 (Feb. 1985), 182-197.

[6] Farina, A., and Galati, G. (1985)

Surveillance radars: State of the art, research, and perspectives.

Alta Frequenza, 54, 4 (1985); reprinted in Radar Applications, M. Skolnik (Ed.), New York: IEEE Press, 1988, 149-166.

[7] Skolnik, M. (1980)

Air surveillance radar.

In Introduction to Radar Systems (2d ed.).

New York: McGraw-Hill, 1980, sec. 14.3.

[8] IEEE Standard Letter Designations for Radar-Frequency

Bands.

IEEE Standard 521, 1984.
[9] Skolnik, M. (2001)

Decorrelation by frequency diversity or frequency agility. In Introduction to Radar Systems (3d ed.).

New York: McGraw-Hill, 2001, 70-71.

[10] O’Donnell, R. M., et al. (1974)

Advanced signal processing for airport surveillance radars.

IEEE EASCON 77, Washington, DC, Oct. 1974, 71A-71F.

[11] Hansen, V. G., and Ward, H. R. (1972)

Detection performance of the cell averaging LOG/CFAR receiver.

IEEE Transactions on Aerospace and Electronic Systems, AES-8 (Sept. 1972), 648-652.

[12] Caputi, W. J. (1971)

Stretch: A time-transformation technique.

IEEE Transactions on Aerospace and Electronic Systems, AES-7 (Mar. 1971), 269-278.

[13] Van Brunt, L. B. (1982)

Applied ECM, vol. 2.

Dunn Loring, VA: EW Engineering, Inc., 1982.

[14] Murrow, D. J. (1990)

Height finding and 3D radar.

In M. Skolnik (Ed.), Radar Handbook.

New York: McGraw-Hill, 1990, ch. 20.

[15] Skolnik, M. (1980)

Height finder and 3D radars.

In Introduction to Radar Systems ( $2 \mathrm{~d}$ ed.).

New York: McGraw-Hill, 1980, sec. 14.4.

[16] Teti, J. G. (2000)

Wide-band airborne radar operating considerations for low-altitude surveillance in the presence of specular multipath.

IEEE Transactions on Antennas and Propagation, 48 (Feb. 2000), 176-191.

[17] Skolnik, M. (2001)

Introduction to Radar Systems (3rd ed).

New York: McGraw-Hill, 2001, eq. 8.4.

[18] Blake, L. V. (1980)

Radar Range-Performance Analysis.

Lexington, MA: Lexington Books, 1980, Fig. 5.3.

[19] Linde, G. J.

Low elevation height finding with a wideband radar. Unpublished manuscript.

[20] Linde, G. J., and Platis, C. V. (1995)

Target recognition with surveillance radar. NRL Review, Naval Research Laboratory, Washington, DC, (1985), 118-120.

[21] Linde, G. J. (2000)

Use of wideband waveforms for target recognition with surveillance radars.

In Record of the IEEE 2000 International Radar

Conference, Alexandria, VA, 128-133.

[22] Hudson, S., and Psaltis, D. (1993)

Correlation filters for aircraft identification from radar range profiles.

IEEE Transactions on Aerospace and Electronic Systems, 29 (July 1993), 741-748.

[23] Zyweck, A., and Bogner, R. E. (1996)

Radar target classification of commercial aircraft.

IEEE Transactions on Aerospace and Electronic Systems, 32 (Apr. 1996), 598-606. 

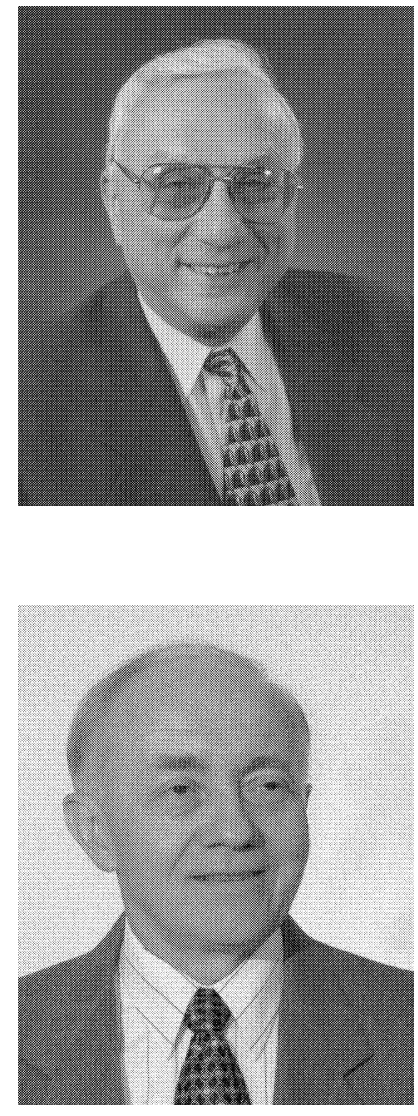

Merrill Skolnik (S'47-A'50-M'55-SM'56-F'69-LF'93) served as Superintendent of the Radar Division at the Naval Research Laboratory, a Senior Executive Service position, from 1965 to 1996. Before that he was with the Institute for Defense Analyses, Research Division of Electronic Communications Inc., MIT Lincoln Laboratory, Sylvania Boston Engineering Laboratory, and The Johns Hopkins University Radiation Laboratory.

Dr. Skolnik has received the IEEE Centennial Medal, the Harry Diamond Award, and the Heinrich Hertz Premium from the Institute of Electronic and Radio Engineers (UK). He has taught a graduate course in radar since 1956, from which evolved the text Introduction to Radar Systems. He is the editor of the Radar Handbook, former editor of the Proceedings of the IEEE, and the author of the entry on radar in the Encyclopedia Britannica. In 1986, Dr. Skolnik was elected to the National Academy of Engineering.

George J. Linde (M'68) received the B.E.E. degree from the University of Minnesota, Minneapolis, in 1965 and the M.S.E.E. from the University of Maryland, College Park, in 1971.

Since 1966 he has been employed by the Radar Division of the Naval Research Laboratory where he has worked in the areas of phased-array radar, low-elevation angle tracking, low probability of intercept radar, shipboard air-surveillance radar, target recognition, and millimeter-wave radar. He was in charge of the development and testing of the high-power, experimental version of the Senrad radar system.

Keith Meads received the B.S. in physics from the University of North Carolina, Charlotte, before joining the Radar Division of the Naval Research Laboratory in 1951.

In the Search Radar Branch he served as Head of the Transmitting Systems Section, Coordinator for Electronic Scanning Systems, and Coordinator for Surveillance Radar which included being the Principal Investigator for the Senrad Exploratory Development Project. He then became Branch Head of the Target Characteristics Branch engaged in radar cross section measurements of aircraft, helicopters, ships, rain and sea clutter; as well as signal processing for pulse compression and ECCM. He was a major contributor as a member of the study group that defined the Aegis weapon system, and the committee that selected the approach used in the Patriot Air Defense System. In 1985, upon retirement from NRL, he was employed as an Advisory Engineer at Westinghouse Electric Corporation in Baltimore in a radar systems engineering group involved with conceptual radar system synthesis and analysis. 